\title{
THE RADIO CHANNEL IN THE VEHICULAR IDENTIFICATION
}

\author{
Ricardo Meneses*, Roberto Linares, Laura Montes \\ Instituto Politécnico Nacional \\ Escuela Superior de Ingeniería Mecánica y Eléctrica, Campus Zacatenco \\ U.P.A.L.M. Edif. 5, Jefatura de ICE, Col. Lindavista, C. P. 07738, México, D. F. \\ *Corresponding author: rmenesesg@ipn.mx \\ Received 14 August, 2010; Revised 11 October, 2010
}

\begin{abstract}
The Radio Frequency Identification System, a wireless communication system, recently it is being applied to vehicular identification, the radio electric and geometry environment characteristics influence the Radio Channel, which one, have been analyzed extensively in different papers, but no one of them has analyzed the RFID technology applied to vehicular technology. The radio channel model is necessary for the effective design of transmitters and receivers, and to determining the positions of the tag and the reader. This motivates the analysis of the propagation environment for outdoor RFID systems. In order to know a feasible RFID application to the vehicular identification, a set of tests were implemented in laboratory and in the field, in order to obtain a channel model that predicts the average power level, a semi-stochastic model, where the average power level is given by the distance raised to some power that depends on the environment, and the field strength in an area around a transmitter, and take into account the structure and materials used at every location in the area. As a result from these tests, the RFID signal and the RFID channel behavior are the focus and discussion of this article.
\end{abstract}

Key words: Reader, tag, electromagnetic environment, backscatter signal, RFID channel.

\section{INTRODUCTION}

The Radio Frequency Identification System, a wireless communication system, is a typical point to point communication system, which one, the radio link formed between the reader, working at the same time as base station and as an end user site, and the tag, the intermediate site, is exposed to electromagnetic disturbances, due reflections off objects in the environment.

The RFID technology objective applied to the vehicular identification is to have all the motor vehicle information, for example, owner name, license, fines, etc. It is a radio mobile typical application, the figure 1 shows the radio channel components, the reader, the tag, the direct ray and the reflected ray from the surface, where the reader is installed in a fixed position, strapped on a fixed post, as the transmitter, and the tag is strapped on the motor vehicle, as a mobile receiver and starting the data back transmission to the reader, living side by side with other communication systems, in a complex and violent electromagnetic environment. Making up the propagation media, we have the free space, the reflections from surface [1], [2], this one, made of homogeneous isotropic material with linear properties, the electric permittivity $\mathcal{E}_{r}$, and the magnetic permeability $\mu_{r}$, being $\varepsilon_{r}=5$, for poor ground (dry) relative electric permittivity [3], and $\varepsilon_{r}=15$, for wet ground relative electric permittivity [3], and the reflections from the various obstacles, some of them statics, and/or some of them, in continuous movement. The figure 1 shows the components of the radio channel, the reader, the tag and the radio channel. 
As a result from the tests, the feasibility RFID application to vehicular identification, depends of several variables, as mismatch polarization between the tag antenna and the reader antenna, in vehicular identification this problem is critic, because the scenario's geometry changes frequently and the polarization matching is very difficult to obtain, and it should, moreover, be remembered that the vehicle's windshield angle is not always the same. Others variables are the speed of the mobile, because, the vehicles cross at different speeds by the site where the reader's antenna, and the reflected waves, to the tag antenna, coming from the reflecting surface. This way, in an effort to meet a radio channel model, these parameters must be considered. A radio channel proposal is the objective of this work, based in multipath environment and variables found in the experimental work.

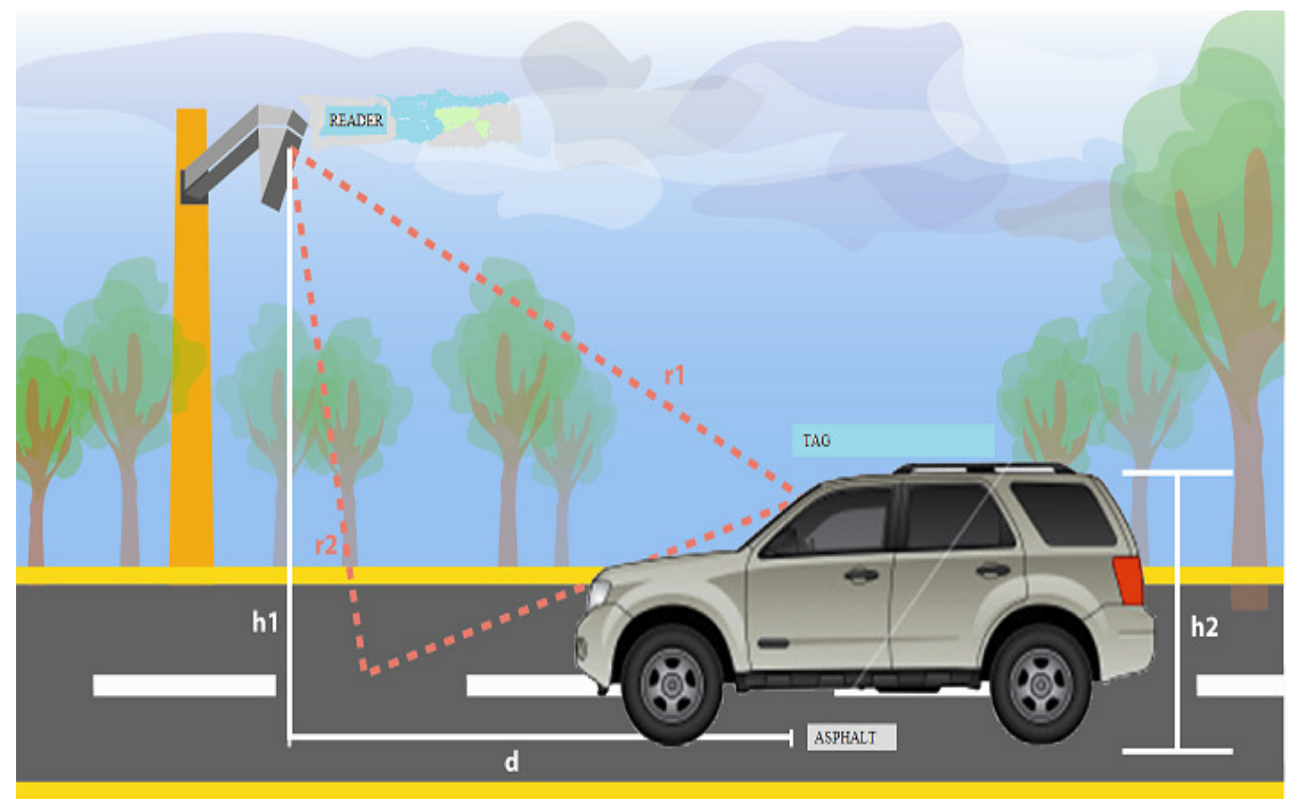

Fig.1.The RFID technology applied to the vehicular identification.

\section{FORMULATION}

\section{The RFID Channel}

Because of the tag draws energy from the forward electromagnetic wave signal transmitted by the reader, part of the electromagnetic wave signal is backscattered/reflected back to the reader by the tag, due to the destructive or constructive contribution, then, the instantaneous mobile antenna received power, can be considered as random variable, which one, depends of the antenna position The backscatter signal is also modulated by the tag through varying the microchip's input impedance in terms of the stored data information to realize backscatter communication, toggling the microchip's input impedance between two discrete states, which produces amplitude shift keying (ASK) or phase shift keying (PSK) impedance modulation. This way, the tag antenna picks up the signal from the reader and the clutter from the obstacles, similarly, the reader picks up the backscattered tag modulated signal and the backscattered modulated clutter signal, too. Then, in the case of the tag, the reader signals and the reflected "signals" enter the receiver, via the tag antenna, to be modulated, as showed in the figure 2. 


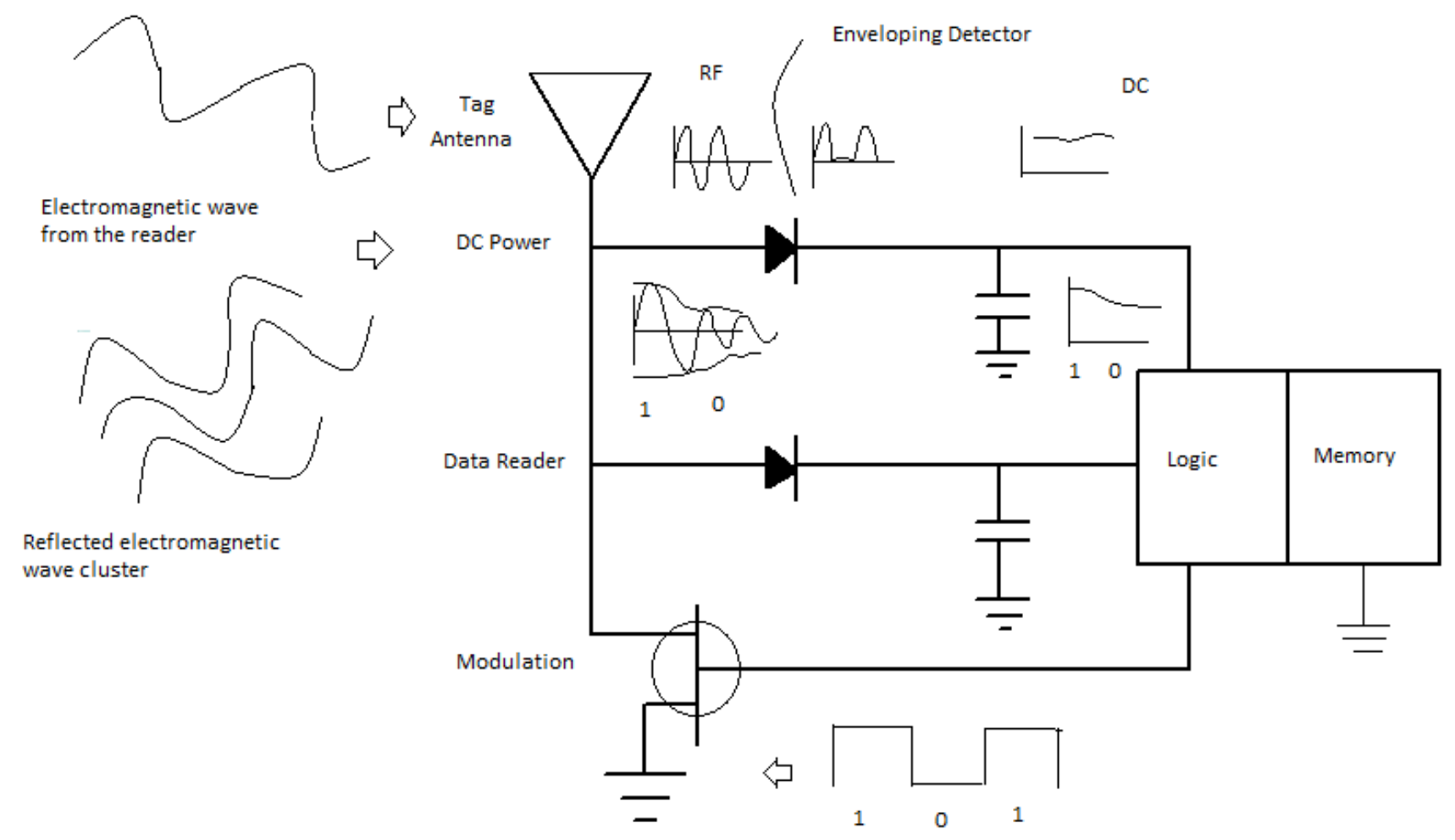

Fig. 2 Tag electronic circuit basic.

Meanwhile, the reader continuously emits RF carrier signals, and keeps observing the received signals for data. The tag modulates the RF field. The data modulation (modulation for $0 \mathrm{~s}$ and $1 \mathrm{~s}$ ) is accomplished by either direct modulation or FSK or Phase Modulation, but, the tag modulates the reflected waves too, as showed in the figure 2. As well as the tag is exposed to multipath fading and variable delays, it is moving as the mobile moves, that is, the tag is involved in a violent electromagnetic environment, where a ray is a direct ray for a while, and an instant later, now, it is a reflected ray. This situation above is showed in the figure 3 , and then, the received signal reader, not only, it is affected by the fading and a constant delay in the RFID channel, indeed, it is affected by multipath fading and variable delays, which one, this situation is represented, with the next expression:

$$
r_{k}(t)=R s_{k}(t+\phi)+n(t)
$$

where:

$R$, Rice or Rayleigh random variable

$\phi$, random variable uniformly between $[-\pi, \pi]$ range

$s_{k}(t)$, input signal between $\left[k T_{s}, k T_{s}+T_{s}\right]$ range

$n(t)$, AWGN signal band limited 


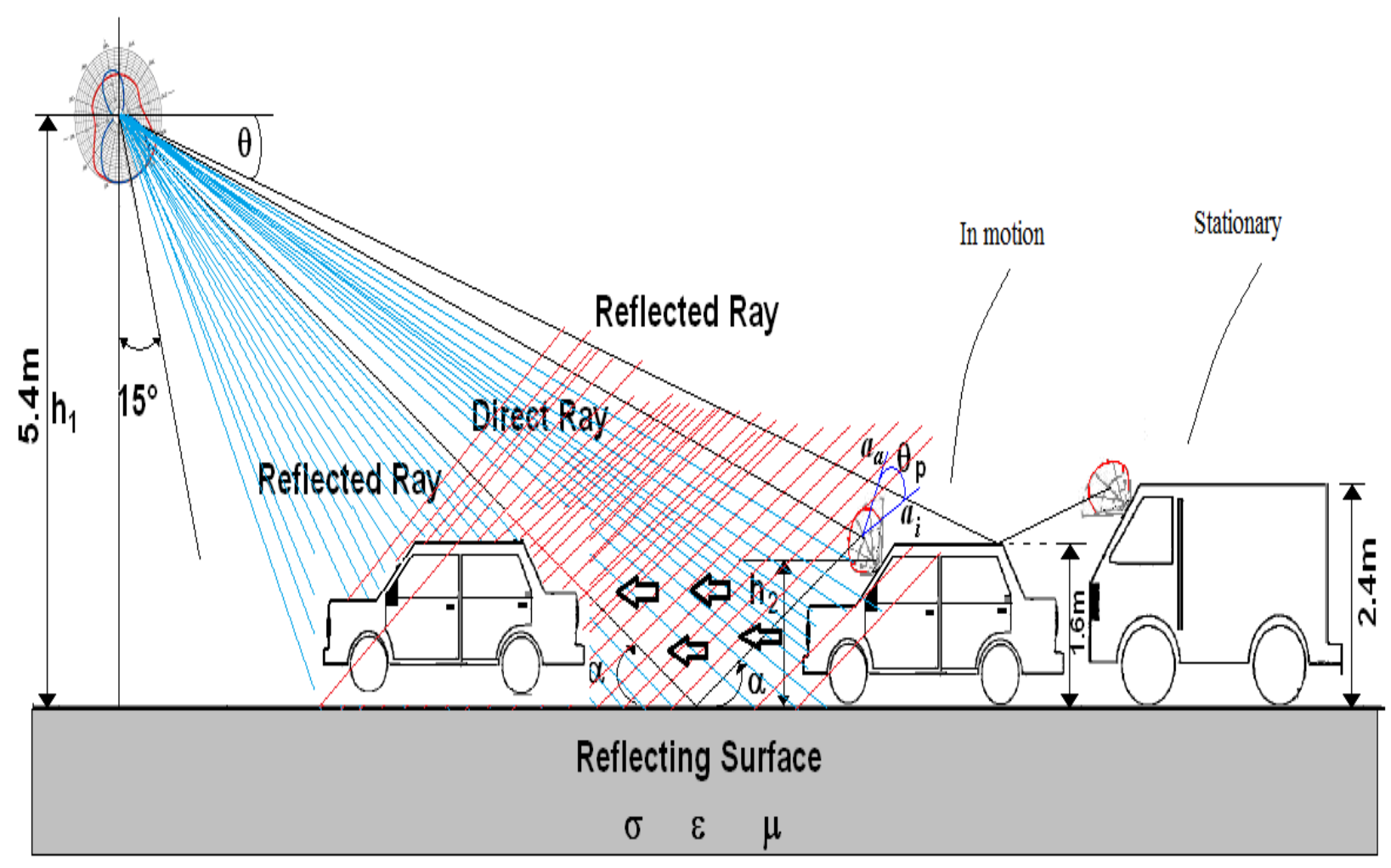

Fig.3. Tag involved in a violent electromagnetic environment.

Starting from (1), if we consider the random variable, $R$, as a Rice variable, it means a multipath environment, where one of multiple paths, it is a dominant path, in this case, there is line of sight (LOS), and the channel is modeled based in the Rayleigh Distribution for the random rays arriving to the tag with different paths, and the dominant path is determined by the path loss models.

In the case where we consider the random variable, $R$, as a Rayleigh variable, there is not a dominant path, but a lot of obstacles are between the reader and the tag, that is, there is not line of sight (NLOS). Like this, if there are obstacles in the transmitter- receiver link, there is a chance to produce time differences that could destroy the received signal. If the time delays between the paths are too long regarding channel bit time length, the signal suffers a great dispersal affecting the bandwidth, that is, a multipath wide band environment.

\section{Modeling the Channel}

Initially, the reader transmits to tag, an unmodulated signal:

$$
S_{R}(t)=A_{D i r} \sin \left(\omega t+\theta_{D i r}\right)
$$

where:

$A_{\text {Dir }}$, no modulated wave signal amplitude

$\theta_{D i r}$, no modulated wave signal phase 
And the modulated backscattered signal from the tag is expressed as follows:

$$
S_{\tau}(t)=A_{\text {Back }} \sin \left(\omega t+\theta_{\text {Back }}\right)
$$

From (3) this signal contains the dominant path and the multipath contributions in amplitude, phase and frequency.

In other hand, the tag modulator section in the tag converts the backscattered/reflected back to the reader, in kth bits block, with, $L=\log _{2} M$, length, described as $x_{L k} \ldots x_{(k+1) L}$, in a analog signal $s_{k}(t)$, then (3) can be expressed as (4), the dominant path, where $d_{k}, q_{k}$, are the amplitudes, and they are dependent on the transmitted energy of the k-th block.

$$
\begin{gathered}
s_{k T->R}(t)=d_{k} \cos \left(2 \pi f_{0} t\right)-q_{k} \operatorname{sen}\left(2 \pi f_{0} t\right) \\
k T_{s}<t<(k+1) T_{s}
\end{gathered}
$$

The figure 4 shows the reader transmitter section and the reader receiver section, the RFID channel, the tag included, which one is affected by noise and several paths.

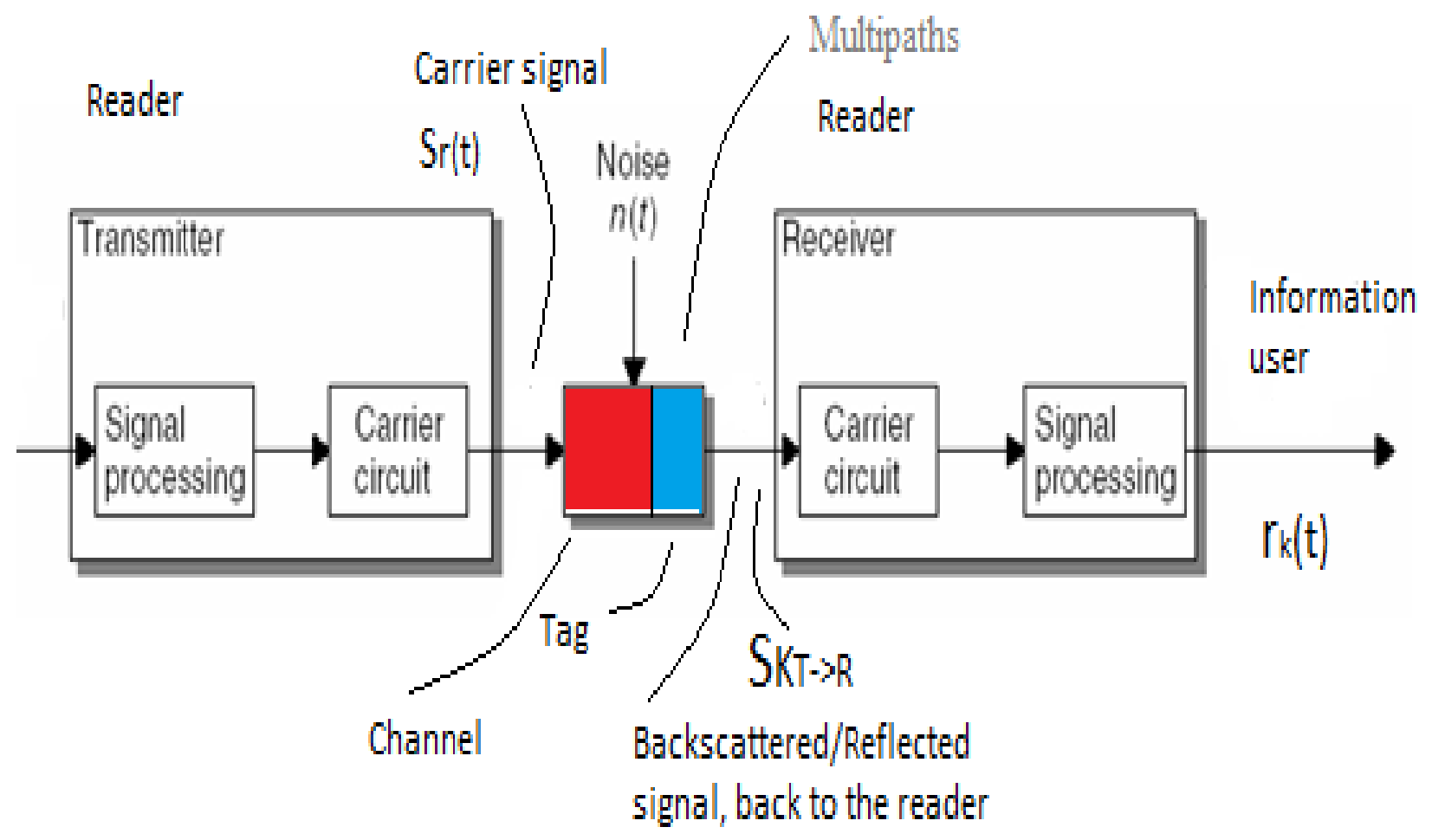

Fig.4. RFID System Model 
If the sources or receiver are moving then the characteristics of the multipaths vary with the time. These time variations are deterministics when the number, location and characteristics of the reflectors are known over time. Otherwise statistical models must be used. Similarly if the number of reflectors is large or if the reflector surfaces are smooth, then we must use statistical approximations to characterize the received signal. The statistics of the relative delay between rays is important because the mean of this delay is directly related to the severity of the dispersive fading and the outage time of the radio system. Thus, the phase displacements result from the motion of the receiver or other spatial change of the receiver location relative to the rest of the propagation environment which may itself including moving objects (reflections from cars and buses, showed in the figure 3). For a mobile receiver, as in our case, the displacement term is given by $\Delta \theta_{n}=(2 \pi v t / \lambda) \cos \left(\alpha_{n}+\phi_{v}\right)$, where $\alpha_{n}$ is the arrival angle of the $n_{t h}$ ray, $v$ is the speed of the motion, and $\phi_{v}$ is the direction of motion, and, due the constructive or destructive contribution of the reflected waves, multipath fading and variable delays, the signal from the tag to the reader, (4) is modified by this situation, and now it is expressed in the form:

$$
\begin{aligned}
& s_{k T->R}(t)=d_{k} \cos \left(\omega_{0} t+\theta\right)-q_{k} \operatorname{sen}\left(\omega_{0} t+\theta\right)+ \\
& \sum_{i=1}^{N} \Gamma(\alpha)_{i}\left[d_{k} \cos \left(\omega_{0} t+\theta_{i}\right)-q_{k} \sin \left(\omega_{0} t+\theta_{i}\right)\right]
\end{aligned}
$$

where:

$\Gamma(\alpha)$, reflection coefficient

$\alpha$, incidence angle

On the other hand, the receiver reader section objective is to enter the received signal $r_{k}(t)$ in the $k T_{s}<t<(k+1) T_{s}$ range, as a $r_{k}=\left[r_{k 1}, r_{k 2}, \ldots r_{k L}\right]$ vector. When a data symbol waveform $u(t)$ is transmitted, in our case FSK or PSK, the resulting received voltage signal is:

$$
r_{k}(t)=\sum_{n=1}^{N} A_{n} u\left(t-\tau_{n}\right) \exp \left(-j\left(\theta_{n}+\Delta \theta_{n}\right)\right)+n(t)
$$

where:

$\tau_{n}$, delay between the rays

$A_{n}$, amplitude of the ray

The expressions (5) and (6) represents an approach to the radio channel model, that, involves the variables affecting the channel, that depends on the environment and take into account the structure and materials (the reflection coefficient) used at every location in the area.

From the several tests made in the field, because the tag is strapped to the motorcar windshield, one of them consists in the mobile tag detection, when the motorcar runs at $40 \mathrm{Km} / \mathrm{h}-60 \mathrm{~km} / \mathrm{h}$ speed, across the antenna reader coverage. 


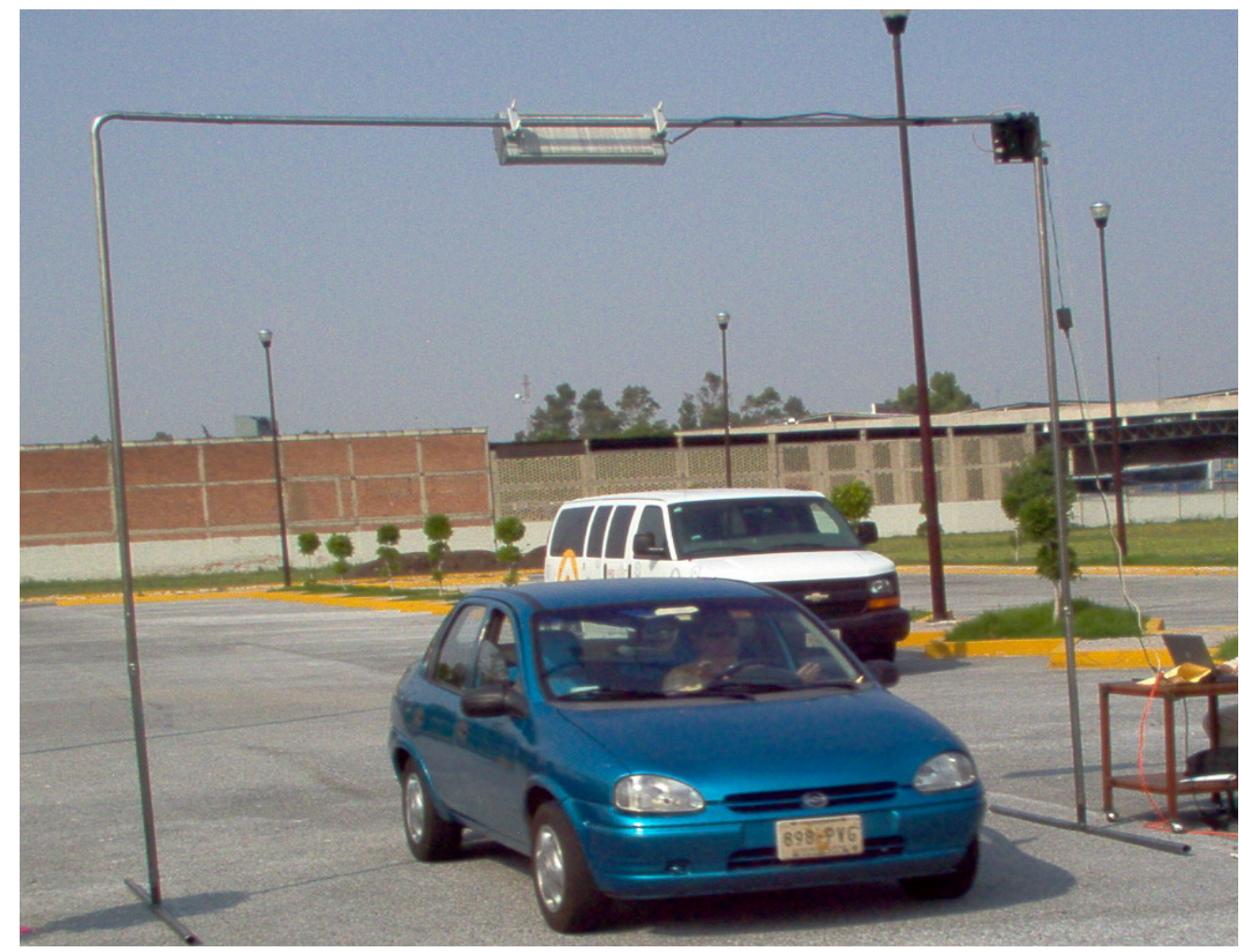

Fig.5. Measurements in the road.

When the mobile speed is greater than $120 \mathrm{~km} / \mathrm{h}$, there is not tag detection, then, the reader is not capable to recover the data. At these speeds, a delay, between rays, exits. The second ray is delayed with respect to the first ray by:

$\tau=\left|\frac{r_{2}-r_{1}}{c}\right|$, where $r_{1}$ and $r_{2}$ are the path lengths of the two rays and $c$ is the propagation velocity.

Finally, from (5), it should be made to clear that, the magnitude and phase of the reflection coefficient are a strong function of the angle of incidence on the reflecting surface (the asphalt), and starting from the two ray model [4,5], and considering the polarization loss factor, the received power is showed in the fig.5 and fig. 6.

The constructive contribution of the reflected waves in the received power (the green curve and the sky blue curve) is showed, where for $\varepsilon_{r}=5$, poor ground (dry) relative electric permittivity [3], the receiver power for vertical polarization is shown in figure 5, as the same way for $\varepsilon_{r}=15$, wet ground relative electric permittivity [3], the receiver power is shown in figure 6. 


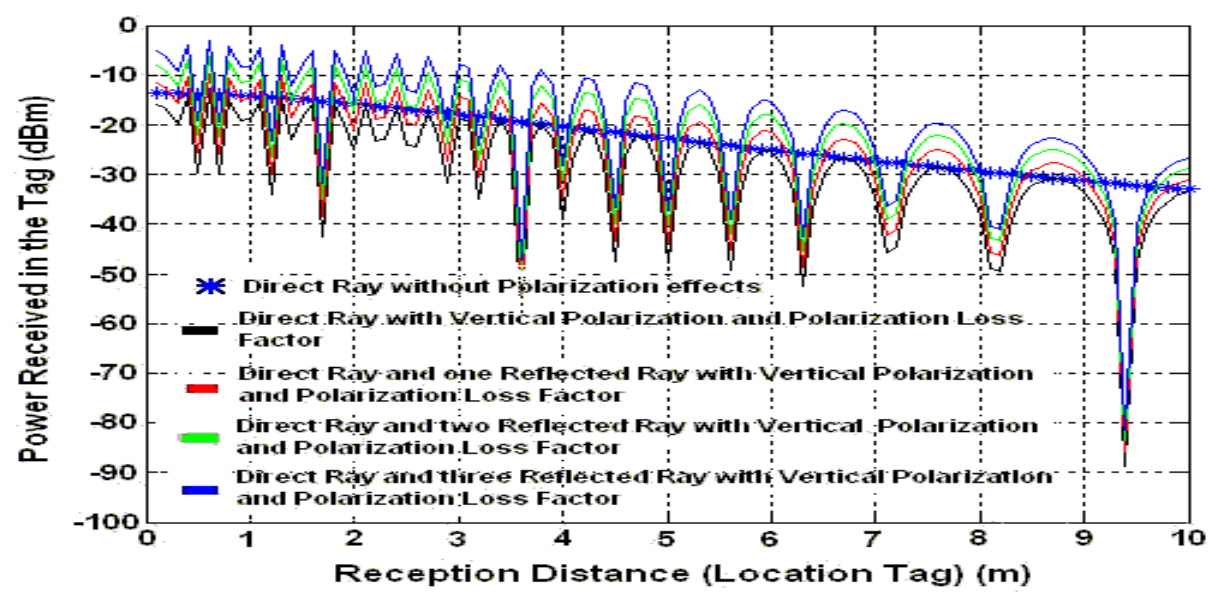

Fig. 5 Tag Received Power with $\varepsilon_{r}=5$ for Vertical Polarization.

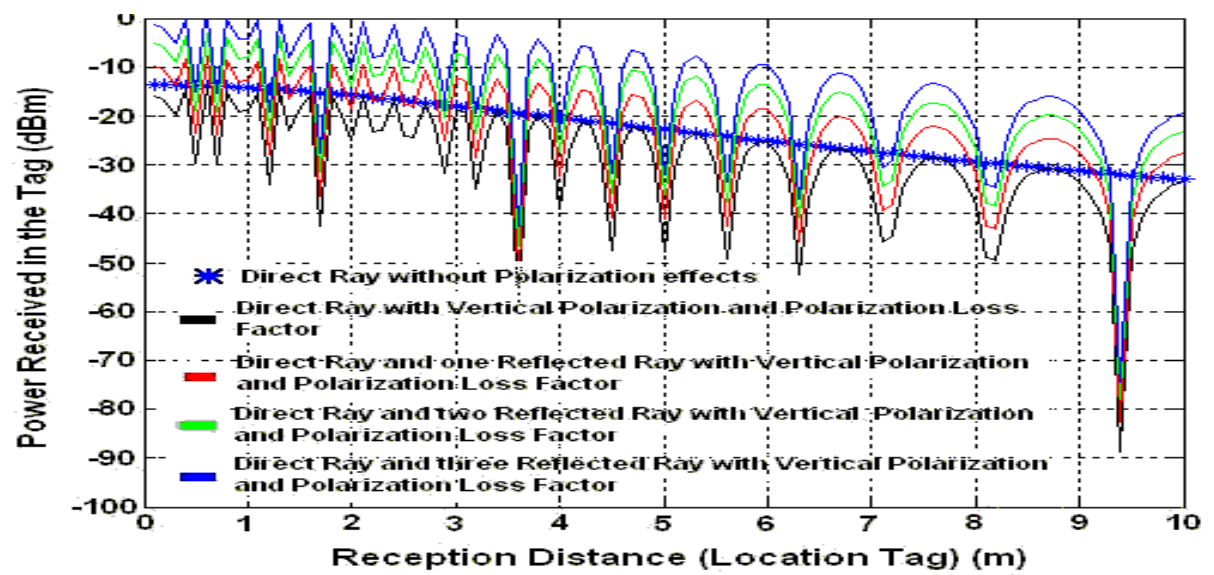

Fig. 6 Tag Received Power with $\varepsilon_{r}=15$ for Vertical Polarization

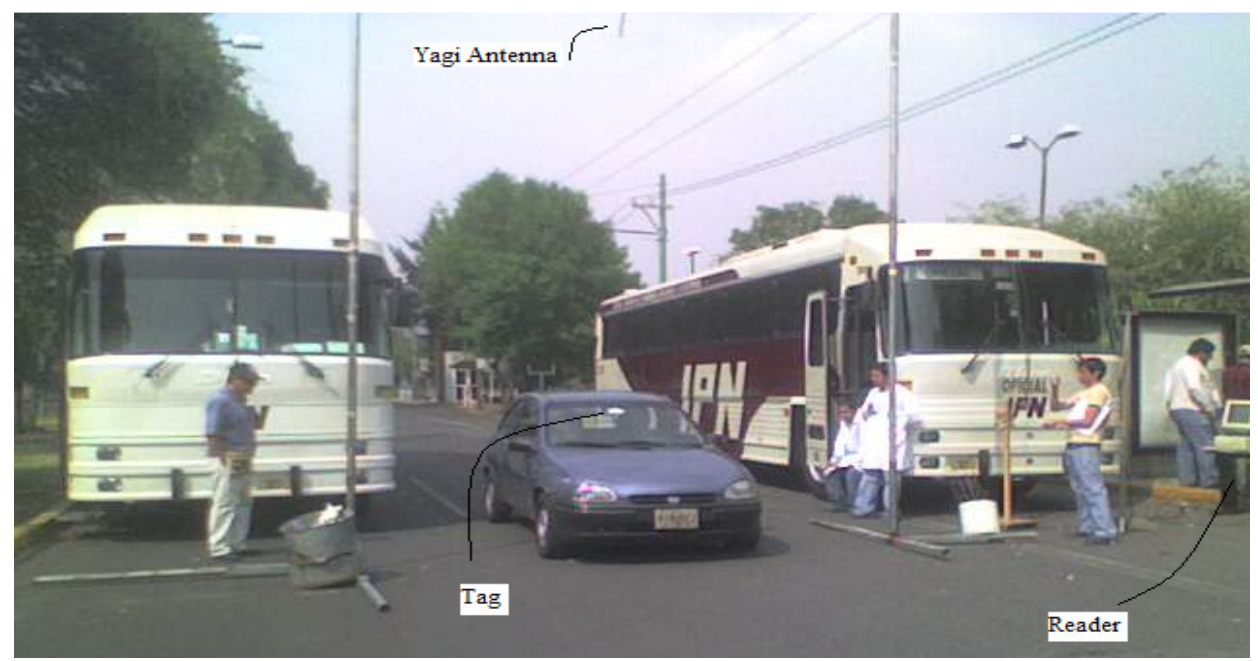

Fig. 7 The buses form a dielectric wall. 
In addition of the tests, the figure 7 shows other scenario, the tag strapped on the motor vehicle under test, the antenna Yagi as receiver-transmitter antenna, the reader and the buses at side, forming a dielectric wall.

\section{CONCLUSION}

As a result from tests, an approach to the radio link behavior is discussed in this work. The ray tracing technique has been used to modeling specific environment, in this case, applied to vehicular technology, where the transmitter is modeled as a source of many rays in all directions around it. Each ray is traced as it bounces and penetrates different objects in the environment including, the ground (asphalt) and walls (buses, vans, sport cars, etc., located at adjacent lanes). Nevertheless, the tracing calculation requires as input a detailed description of the environment, because every point of reflection of each ray from a surface has to be characterized in terms of the surface material and geometry. Polarization mismatch antennas, speed of the mobile, speed of surrounding objects, and the transmission bandwidth of the signal are other important physical factors in the radio propagation channel influence.

\section{REFERENCES}

1. Dolukhanov, M. 1971. "Propagation of Radio Waves”, MIR Publishers, Moscow, USSR.

2. H. S. Kim, R.M. Narayanan, 2002. “A New Measurement Technique for Obtaining the Complex Relative Permitivity of Terrain Surfaces", IEEE Transactions on Geoscience and Remote Sensing.

3. Parsons, J. D. 2000. "The Mobile Radio Propagation Channel”, Second Edition John Wiley \& Sons, England.

4. Anderson, H. A. 1993. "A Ray Tracing Propagation Model for Digital Broadcast Systems in Urban Areas", IEEE Transaction on Broadcasting Vol. 39, Number 3.

5. Xia H.H., Bertoni H.L., Maciel L.R., Lindsay-Stewart A. and Rowe R., 1993. "Radio Propagation Characteristics for Line-of-Sight Microcellular and Personal Communications, IEEE Trans. Antennas Propagat., vol 41, pp. 1439-1446.

6. EPC Global, "Tag Performance Parameters and Test Methods", EPC-global Inc. epcglobal@epcglobalinc.org, version 1.1.2

7. $\quad$ EPC Global, "Specification for RFID air interface Radio Frequency Identity Protocols Class 1 generation 2 UHF RFID Protocol for Communication@860-960 MHz" EPCGlobal Inc. global@epcglobal.org, version 1.0.9.

8 H. Hashemi, 1983. "Impulse response modeling of indoor radio propagation channels", IEE J. selected Areas Commun, vol. 11, no.7.

9. P. V. Nikitin, Rao, K.V.S., 2008. “Antennas and Propagation in UHF RFID Systems”, IEEE RFID 2008 Conference Proceedings (Las Vegas, NV, April 16-17, 2008).

10 Lazaro, A., Girbau D., Salinas D., 2009. "Radio Link Budgets for UHF RFID on Multipath Environments", Antennas and Propagation, IEEE Transactions on, Volume 57, Issue 4. 